\title{
Der Briefwechsel von Leonhard Euler und Christian Goldbach
}

Helmut Koch

\section{Einleitung}

Leonhard Euler ist einer der bedeutendsten Mathematiker aller Zeiten, Christian Goldbach war ein universell gebildeter Wissenschaftler, der eine wichtige Rolle in der Petersburger Akademie der Wissenschaften spielte.

Der Briefwechsel zwischen ihnen umfasst 102 Briefe von Euler und 94 Briefe von Goldbach. Er ist wichtig im Rahmen des Gesamtwerkes von Euler und für die Wissenschaftsgeschichte. Er wurde zuerst 1843 von Paul Heinrich Fuss [4], einem Urenkel von Euler, publiziert. Diese Publikation war jedoch unvollständig. Eine vollständige, kommentierte Publikation erschien 1965 im Akademie-Verlag Berlin [6]. Dabei sind die Briefe durchnummeriert von 1 bis 196. Im folgenden werden die Briefe entsprechend dieser Nummerierung zitiert.

\section{Zur Biografie von Christian Goldbach}

Die Biografie von Euler ist wohlbekannt. Wir verweisen dazu auf den vorhergehenden Artikel von Norbert Schappacher [10] und die dort genannte Literatur. Zur Biografie von Goldbach liegen weniger Quellen vor. Es gibt vor allem seine unpublizierten Tagebuchaufzeichnungen, die seiner Lebensbeschreibung durch A.P. Jushkevich und Ju.Kh. Kopelevich [5] zugrunde liegen.

Christian Goldbach wurde am 18. März 1690 in Königsberg in Ostpreußen geboren. Sein Vater war evangelischer Geistlicher und Professor für Geschichte und Rhetorik an der Universität Königsberg.

1706 beginnt Christian Goldbach das Studium der Jurisprudenz an dieser Universität. Jedoch interessiert er sich auch für Mathematik, Poesie und vieles andere. Im Frühjahr 1709 begibt er sich auf Reisen zunächst innerhalb von Ostpreußen. Den Herbst 1710 verbringt er in Frankfurt an der Oder, wo er seine juristischen Studien an der Universität vervollständigt. Nach und nach dehnt er seine Reisen auf Deutschland und Europa aus. In den Städten, die er besucht, nimmt er Verbindung zu dort wohnenden Wissenschaftlern 
verschiedener Disziplinen auf und versteht es, diese Verbindung auch nach seiner Abreise durch Briefwechsel aufrecht zu erhalten. Im Jahre 1711 macht er die Bekanntschaft von Leibniz in Leipzig, den er 1712 in dessen Wohnung in Hannover besucht. Im gleichen Jahr verteidigt Goldbach eine Dissertation an der Universität Groningen zum Thema Die Bestrafung von Geiselnehmern in verschiedenen Ländern. Von Dezember 1714 bis Mai 1718 lebt Goldbach wieder in Königsberg. Jedoch haben wir für diese Zeit keine Informationen, da hierzu keine Tagebuchaufzeichnungen vorhanden sind.

Nach und nach baut Goldbach ein Netz von Beziehungen zu bedeutenden Wissenschaftlern in Europa auf. So trifft er 1721 Nicolaus Bernoulli in Venedig und beginnt 1723 einen Briefwechsel mit dessen Bruder Daniel Bernoulli.

1725 erhält das Leben von Goldbach eine entscheidende Wende. Auf Initiative von Peter I. wird in diesem Jahr die St. Petersburger Akademie der Wissenschaften gegründet. Laurentius Blumentrost, der Leibarzt von Peter I., wird mit der Gründung beauftragt und ist ihr erster Präsident. Dieser fragt bei verschiedenen Wissenschaftlern an, wer als Mitglied der neuen Akademie in Frage käme. J.G. Doppelmaier in Nürnberg reicht die Frage weiter an Goldbach, der Nicolaus Bernoulli vorschlägt. Dieser wird zusammen mit seinem Bruder Daniel berufen.

Goldbach selbst macht sich auf den Weg nach St. Petersburg. Von Riga aus schickt er einen Brief an Blumentrost mit der Anfrage, ob er der Akademie nützlich sein könne. Blumentrost antwortet, dass alle Stellen für Mitglieder besetzt seien. Trotz dieser Absage reist Goldbach weiter nach Petersburg. Dort wird er nach drei Tagen von Blumentrost empfangen. Das mit ihm geführte Gespräch ist entscheidend. Goldbach erhält die Stellung eines ständigen Sekretärs der Akademie. Zusammen mit den Brüdern Bernoulli empfiehlt er die Berufung von Euler. Dieser wird zur Arbeit an der Akademie eingeladen und erhält zunächst die Stellung eines Adjunkts der Mathematischen Klasse. Er kommt 1727 im Alter von 20 Jahren nach Petersburg. Im gleichen Jahr wird Goldbach zum Erzieher des Zarewitschs bestimmt, der bald darauf nach dem Tode von Katharina I. als Peter II. Zar wird, jedoch schon 1730 im Alter von 14 Jahren verstirbt. 1728 geht Goldbach mit dem Hof nach Moskau, von wo er 1732 nach Petersburg zurückkehrt.

Am 13. Oktober 1729 schreibt Euler den ersten Brief an Goldbach auf Empfehlung von Daniel Bernoulli.

Nach seiner Rückkehr nach Petersburg übernimmt Goldbach den Vorsitz bei den Sitzungen der Akademie bis er im Jahre 1742 als Staatsrat in das Kollegium für auswärtige Angelegenheiten berufen wird. Er bleibt jedoch Ehrenmitglied der Akademie bis zu seinem Tode am 1. Dezember 1764.

\section{Die Goldbach-Vermutung}

Vielen Mathematikern ist Goldbach heute bekannt durch die Goldbach-Vermutung, die er im Brief vom 27. Mai 1742 wie folgt ausspricht:

Es scheint wenigstens, dass eine jede Zahl, die größer als 2, ein aggregatum trium numerum primorum sei. Die unitatem mit dazugerechnet.

D.h. jede natürliche Zahl größer als 2 ist Summe von drei Zahlen, die Primzahlen oder gleich 1 sind. 
Heute unterscheidet man die Ternäre und die Binäre Goldbach-Vermutung.

Die Ternäre Goldbach-Vermutung besagt, dass sich jede ungerade Zahl größer als 5 als Summe von drei Primzahlen darstellen lässt. Hierzu gibt es das grundlegende Ergebnis von I.M. Vinogradov aus dem Jahre 1937, dass jede genügend große ungerade Zahl $n$ Summe von drei Primzahlen ist [11]. Heute weiß man, dass jedes $n$ mit $n>6 \cdot 10^{7193}$ Summe von drei Primzahlen ist. Mit Computern hat man das auch für $n<1,13256 \cdot 10^{22}$ berechnet.

Die Binäre Goldbach-Vermutung besagt, dass jede gerade Zahl größer als 2 Summe von zwei Primzahlen ist. Die vorliegenden Ergebnisse zu dieser Vermutung sind schwächer als im ternären Fall. Man hat folgendes Ergebnis: Sei $E(x)$ die Anzahl der geraden Zahlen, die kleiner oder gleich einer Schranke $x$ sind, und sich nicht als Summe von zwei Primzahlen darstellen lassen. Dann gilt $E(x)<x^{1-\delta}$ für ein gewisses $\delta>0$. Insbesondere gilt

$$
\lim _{x \rightarrow \infty} \frac{E(x)}{x}=0
$$

\section{Verlauf des Briefwechsels}

Der Briefwechsel zwischen Euler und Goldbach beginnt mit einem Brief von Euler am 13. Oktober 1729 alten Stils, d.h. nach dem zu dieser Zeit in Russland gültigen Julianischen Kalender. In Deutschland gilt damals wie heute der Gregorianische Kalender. Man spricht in diesem Fall von neuеm Stil. Die Datierung der Briefe ist uneinheitlich. Im folgenden werden Briefe aus Russland im alten und aus Deutschland im neuen Stil datiert. Der Unterschied beträgt im 18. Jahrhundert 11 Tage.

Zum Zeitpunkt des ersten Briefes ist Euler in St. Petersburg und Goldbach als Erzieher des minderjährigen Zaren in Moskau. Der unverheiratete Euler wohnt mit Daniel Bernoulli in einer Wohnung. Dieser hatte in den Jahren 1728/29 einen ausgedehnten Briefwechsel mit Goldbach über Reihenprobleme und insbesondere die Interpolation der Fakultät $x$ !. Am Anfang seines Briefes sagt Euler, dass er auf Empfehlung von Bernoulli schreibt und nimmt Bezug auf den Briefwechsel Bernoulli-Goldbach. Euler gibt in seinem Brief für $x$ ! eine Integraldarstellung an. Nach Legendre bezeichnet man diese Funktion heute mit $\Gamma(x+1)$.

Die ersten 32 Briefe sind im Zeitraum 1729-1739 in Latein, der damaligen Wissenschaftssprache, geschrieben. Sie haben rein mathematischen Inhalt. Der 33. Brief ist eine Gratulation Goldbachs zum Abschluss eines neuen Vertrages von Euler mit der Petersburger Akademie. Dieser Brief ist französisch geschrieben. Alle folgenden Briefe sind auf deutsch abgefasst, wobei in mathematischen Darstellungen oft aufs Lateinische zurückgegriffen wird, sodass eine deutsch-lateinische Mischsprache entsteht.

Der Brief 184 von Euler aus Berlin ist auf den 26. April 1757, der nächste Brief auf den 29. Juni 1762 datiert. Diese Lücke erklärt sich durch den Siebenjährigen Krieg, in dem Preußen und Russland Kriegsgegner waren. Der letzte Brief, wiederum von Euler, stammt vom 17. März 1764. Hierauf ist keine Antwort Goldbachs bekannt, der am 1. Dezember 1764 verstarb. 


\section{Zur Zeit- und Wissenschaftsgeschichte}

Wenn auch der Hauptinhalt der Briefe die Mathematik betrifft, werden doch auch zahlreiche andere Fragen berührt. Wir beginnen mit Ausführungen zur Zeit- und Wissenschaftsgeschichte. Im Brief 36 vom 18. April 1741 spricht Euler über den Beginn des ersten Schlesischen Krieges zwischen Preußen und Österreich, den Preußen mit einem Überfall auf Niederschlesien ausgelöst hatte:

Der H. Delisle geht diesen Augenblick von mir weg und hat mir gesagt, dass bei Neuss schon wirklich eine blutige Bataille vorgegangen, wobei von preuBischer Seite zwei Prinzen nebst einige Generalen, von österreichischer Seite aber der General Lentulus nebst noch vielen anderen geblieben; doch endlich aber die Preußen das Feld behalten haben. Auch soll Mr. Maupertuis, welcher von dem König Abschied nehmen wollen, bei dieser Aktion verloren gegangen sein.

Euler schreibt Neuss meint aber die schlesische Stadt Neiße. Heute wird die Schlacht genauer nach dem Ort Mollwitz benannt. Joseph Nicolas Delisle (1688-1768) war ein französischer Astronom, Mitglied der Petersburger Akademie. Pierre-Louis Moreau de Maupertuis (1698-1759) war von Friedrich II. mit der Neuformierung der Berliner Akademie der Wissenschaften beauftragt. Maupertuis geriet während der Schlacht bei Mollwitz in Österreichische Gefangenschaft. Er wurde jedoch in Wien vom kaiserlichen Hof ehrenvoll empfangen und dann wieder nach Berlin entlassen, wohin er allerdings erst 1745 zurückkehrte. Die Akademie nahm 1743 ihre Arbeit auf.

Der Brief 38 vom 1. August 1741 ist der erste Brief Eulers nach seiner Übersiedlung nach Berlin. Er enthält eine ausführliche Beschreibung seiner Schiffsreise von St. Petersburg nach Stettin vom 19. Juni bis 13. Juli 1741 sowie seiner Reise mit Pferdekutsche von Stettin nach Berlin vom 22. Juli bis 25. Juli 1741.

Im Brief 52 schreibt Euler am 30. Juni 1742 über das Ende des ersten Schlesischen Krieges:

Allhier lebt nun alles in den größten Freuden, da heute der schon längst gewünschte Friede zwischen unserm allergnädigsten König und der Königin von Ungarn und Böheim mit allen Solennitäten publiziert worden.

Weiter schreibt Euler zu seinem fortbestehenden Engagement bei der Petersburger Akademie:

Vor einigen Posttagen habe ich von Petersburg die völlige Resolution wegen meines Engagements bei der Akademie nebst einer jährlichen Pension von 200 Rub. erhalten, und über dieses ist mir noch die Restitution aller auf die Korrespondenz gehenden Unkosten versprochen worden.

In den 24 Jahren seines Aufenthalts in Berlin hat er mehr als 100 Arbeiten nach St. Petersburg geschickt.

In seinem Brief 72 vom 24. August 1743 berichtet Euler über die Eröffnung der neuen Akademie: 
Ew. Wohlgeb. werden ohne Zweifel schon vernommen haben, dass die neue Sozietät der Wissenschaften allhier den $1^{\text {ten }}$ dieses Monats ihren Anfang genommen und dass die beiden HH. Cabinetsministres Graf von Podewils und von Bork, wie auch der H. Generalfeldmarschall von Schmettau sich nicht nur zu Mitgliedern erklärt, sondern auch den Versammlungen fleißig beiwohnen.

Im Brief 80 vom 4. Juli 1744 schreibt Euler über die Sprache der Berliner Akademie:

Die neue Akademie allhier wird nächstens einen Tomum von den darin abgelesenen Piecen herausgeben. Es wird darin eine große Anzahl Piecen von mir kommen. Weilen nun die H. Staatsministri fleißig zugegen sind, so habe, um diesen Herren keinen Ekel zu erwecken, meine Dissertationen französisch abgelesen, nachdem solche von dem H. Prof. Naudé korrigiert worden.

Philippe Naudé (1684-1745) war ein Gymnasiallehrer französischer Herkunft, der Euler auch mathematisch angeregt hat, worauf wir im weiteren zurückkommen.

Seine Abneigung gegen die französische Sprache, die er gut beherrschte, äußert Euler noch stärker im Brief 189 vom 1. Oktober 1763:

Noch hat sich hier der Anschein nicht verloren, dass die hiesige Akademie in eine Academie Françoise verwandelt werden soll; so sehr ich mich vor einer nochmaligen Ortsveränderung entsetze, so würde ich mich doch in diesem Fall dazu entschließen müssen, und nichts würde mich dabei herzlicher erfreuen, als Ew. Hochwohlgeb. nochmals sehen zu können.

Man muss diese Äußerung als Wunsch Eulers verstehen, nach St. Petersburg zurückzukehren. Dies geschah 1766, aber Goldbach war schon 1764 gestorben.

Als letzte Bemerkung zum Leben von Euler erwähnen wir seine Verbindung zu dem baltischen Adligen Hermann Graf von Keyserling (1697-1765), der 1733 Präsident der Petersburger Akademie, später Botschafter Russlands in Polen, Sachsen und Preußen war. Im Brief 117 vom 4. Juli 1747 schreibt Euler:

In meiner Familie hat sich seit dem nichts Veränderliches zugetragen, als dass neulich meine Frau wiederum mit einem Söhnlein niedergekommen, welches H. Graf von Kaiserling aus der Tauf gehoben und Herman Friedrich genannt.

Keyserling gab 1741 bei Johann Sebastian Bach die Goldberg-Variationen in Auftrag. So ergibt sich ein Zusammenhang zwischen Euler und Bach, den vielleicht produktivsten Genien aller Zeiten.

\section{Mathematische Anregungen für Euler durch Goldbach}

Die wichtigste Anregung durch Goldbach findet sich im Brief 2 vom 1. Dezember 1729. In einem Postscriptum merkt Goldbach an, dass die Behauptung Fermats, dass $2^{2^{n}}+1$ für alle natürlichen Zahlen $n$ eine Primzahl ist, nicht bewiesen sei. Diese Behauptung war bekannt für $n \leq 4$ aber unbekannt für $n>4$. 
Für Euler ist dieser Brief eine Anregung, Fermat zu lesen, wovon er im Brief 5 vom 4. Juni 1730 schreibt. Ihn begeistert die Behauptung Fermats, dass sich jede natürliche Zahl als Summe von höchstens vier Quadraten darstellen lässt. Mit dieser Behauptung hat sich Euler sein Leben lang beschäftigt. Endgültig wurde sie allerdings erst von Lagrange gelöst [8], der auf Eulers Ergebnisse aufbaut.

Was die Frage anbelangt, unter welchen Bedingungen $2^{a}+1$ eine Primzahl ist, kann man zunächst anmerken, dass dazu $a$ eine Zweierpotenz sein muss. Wenn $a$ einen ungeraden Faktor $b$ hat, so ist $2^{a / b}+1$ ein Teiler von $2^{a}+1$ entsprechend der allgemeineren Formel

$$
x^{b}-y^{b}=(x-y)\left(x^{b-1}+x^{b-2} y+\cdots+y^{b-1}\right)
$$

angewandt auf $x=2^{a / b}, y=-1$.

Euler findet, dass 641 ein Teiler von $2^{2^{5}}+1$ ist. Dabei benutzt er den Satz, dass jeder Primteiler $p$ von $2^{2^{n}}+1$ die Form $2^{n+1} m+1$ für ein gewisses $m$ hat. Heutzutage beweist man diesen Satz gruppentheoretisch: Aus $2^{2^{n}} \equiv-1(\bmod p)$ folgt $2^{2^{n+1}} \equiv 1(\bmod p)$, und es gibt keine natürliche Zahl $c$ mit $c<2^{n+1}$ und $2^{c} \equiv 1(\bmod p)$. Daher ist $2^{n+1}$ ein Teiler der Ordnung der Gruppe der primen Restklassen modulo $p$. Das ergibt die Behauptung des Satzes. Eulers Argumentation entspricht diesem Beweis. Die Eulerschen Überlegungen können als erste Anfänge der Gruppentheorie verstanden werden.

Eulers Gegenbeispiel ergibt sich aus dem Satz für $n=5, m=10$. Bis heute hat man keine natürliche Zahl $n>4$ gefunden, für die $2^{2^{n}}+1$ eine Primzahl ist. Man weiß, dass $2^{2^{n}}+1$ für $5 \leq n \leq 32$ keine Primzahl ist. Euler publizierte sein Gegenbeispiel 1732 in [2].

Nach dem Tode von Fermat im Jahre 1665 kam das Interesse an zahlentheoretischen Fragen zum Erliegen. Erst Euler nimmt auf Anregung von Goldbach das Thema wieder auf. Es spielt im Briefwechsel der beiden eine Hauptrolle.

Weniger direkt sind die Anregungen Goldbachs im Falle der Untersuchung der Funktion

$$
\zeta(s):=\sum_{n=1}^{\infty} \frac{1}{n^{s}},
$$

die heute als Riemannsche Zetafunktion bezeichnet wird, da Riemann [9] eine tiefliegende Untersuchung der Funktion in Zusammenhang mit der Primzahlverteilung durchgeführt hat.

Schon Leibniz und später die Bernoulli-Brüder haben sich für die Frage der Werte von $\zeta(m)$ für natürliche Zahlen $m \geq 2$ interessiert. Euler löste dieses Problem für gerade $m$ vollständig. 1735 zeigte er $\zeta(2)=\frac{\pi^{2}}{6}$ und 1739 allgemein

$$
\zeta(2 n)=(-1)^{n-1} \frac{B_{2 n}}{2[2 n] !}(2 \pi)^{2 n},
$$

wobei $B_{m}, m=0,1, \ldots$, die Bernoullizahlen bezeichnen, die rekursiv durch

$$
B_{0}=1, \sum_{k=0}^{m}\left(\begin{array}{c}
m+1 \\
k
\end{array}\right) B_{k}=0
$$

definiert sind. 
Im Brief 26 vom 27. Oktober 1739 gibt Goldbach die Reihe

$$
\sum_{n=1}^{\infty} \lambda(n) \frac{1}{n^{m}}
$$

an mit $\lambda(n)=1$ bzw. -1 je nachdem, ob $n$ Produkt einer geraden oder ungeraden Anzahl von Primzahlen ist. Ohne Beweis gibt er an, dass diese Reihe für gerade $m \geq 2$ gleich $\alpha \pi^{m}$ sei mit rationalem $\alpha$.

Im Brief 27 vom 12. November 1739 zeigt Euler, dass diese Reihe gleich $\frac{\zeta(2 m)}{\zeta(m)}$ ist, woraus Goldbachs Behauptung nach dem oben genannten Ergebnis von Euler über $\zeta(m)$ folgt.

In einer Anmerkung zu Goldbachs Brief 28 vom 24. November 1739 notiert Euler die Gleichung

$$
\sum_{n+1}^{\infty} \mu(n) \frac{1}{n^{s}}=\frac{1}{\zeta(s)},
$$

wobei $\mu(n)$ die Moebiussche Funktion bezeichnet. Dieses Ergebnis wurde erst 1748 in Eulers Introductio in Analysin Infinitorum publiziert.

In einigen Fällen enthalten die Briefe von Goldbach auch elementare Fehler, die dann von Euler korrigiert werden.

Im Brief 6 vom 15. Juni 1730 stellt Goldbach die Behauptung auf, dass der kleinste Teiler von $a^{2}+1$, der größer als 1 ist, wieder von der Form $b^{2}+1$ ist, wobei $a$ und $b$ natürliche Zahlen sind. Im Brief 7 gibt Euler das Gegenbeispiel $a=34$ mit 13 teilt $a^{2}+1$ an.

Im Brief 49 vom 12. April 1742 meint Goldbach, dass $(a+b)^{p}-a^{p}-b^{p}$ für eine natürliche Zahl $p$ immer durch einen Primteiler von $p$ teilbar sei. Im Brief 50 gibt Euler das Gegenbeispiel $a=b=1, p=35$, an.

\section{Der Zweiquadratesatz}

$\mathrm{Zu}$ den berühmten Vermutungen von Fermat, die von Euler bewiesen wurden, gehört der Zweiquadratesatz:

(i) Jede Primzahl p der Form $4 n+1$ ist in eindeutiger Weise als Summe zweier Quadrate darstellbar.

(ii) Die Summe zweier Quadrate relativ primer Zahlen hat keinen Faktor der Form $4 n-1$.

Dieser Satz wird in einer Reihe von Briefen angesprochen. Eulers erster Beitrag hierzu ist sein Beweis von (ii), den er im Brief 47 vom 6. März 1742 Goldbach mitteilt:

Angenommen die Primzahl $p=4 n-1$ teilt $a^{2}+b^{2} \operatorname{mit} \operatorname{ggT}(a, b)=1$. Dann folgt, dass $p$ auch $a^{2(2 n-1)}+b^{2(2 n-1)}$ teilt. Andererseits teilt $p$ aber $a^{4 n-2}-b^{4 n-2}$ nach dem Fermatschen Satz, dass $x^{p-1} \equiv 1(\bmod p)$ für alle zu $p$ primen ganzen Zahlen $x$ ist. Es folgt, dass $p$ ein Teiler von $a$ und $b$ ist im Widerspruch zur Voraussetzung. 
Weitere Mitteilungen zum Zweiquadratesatz finden sich in Eulers Briefen 52, 87, 115, 125 und 138. Im Brief 138 vom 12. April 1749 kann Euler endlich mitteilen:

Nunmehro habe ich endlich einen bündigen Beweis gefunden, dass ein jeglicher numerus primus von dieser Form $4 n+1$ eine summa duorum quadratorum ist.

Nachdem, was Euler bereits in vorhergehenden Briefen bewiesen hatte, genügte es, den folgenden Satz zu beweisen:

Sei $p=4 n+1$ eine Primzahl. Dann gibt es eine natürliche Zahl a mit $p \mid\left(a^{2 n}+1\right)$.

Eulers Beweis verläuft folgendermaßen: Wegen $x^{4 n} \equiv 1(\bmod p)$ für zu $p$ prime ganze Zahlen $x$ ist $p$ ein Teiler von $\left(x^{2 n}-1\right)\left(x^{2 n}+1\right)$. Zum Beweis des Satzes genügt es also, die Annahme $p \mid\left(x^{2 n}-1\right)$ für alle $x$ mit $1 \leq x \leq 4 n$ zum Widerspruch zu führen. Euler erreicht das in folgender Weise:

Die Annahme hat zur Folge, dass $p$ ein Teiler der Differenzen $2^{2 n}-1,3^{2 n}-2^{2 n}, \ldots$, $(4 n)^{2 n}-(4 n-1)^{2 n}$ ist. Entsprechendes gilt für die zweiten Differenzen usw. Aber die $2 n$-ten Differenzen sind alle gleich $(2 n)$ !. In der Tat, setzt man $f(a)=a^{m}$ und $(D f)(a)=$ $(a+1)^{m}-a^{m}$, so ist $(D f)(a)$ ein Polynom vom Grade $m-1$ in $a$ mit Koeffizienten $m$ bei $a^{m-1}$. Es folgt $\left(D^{(m)} f\right)(a)=m$ ! für die $m$-te Differenz.

Man beweist heute, Lagrange [7] folgend, diesen Satz viel einfacher mit dem Hinweis, dass ein Polynom vom Grade $m$ nicht mehr als $m$ Nullstellen haben kann. Das gilt für jeden Körper. Hier handelt es sich um den Körper mit $p$ Elementen.

\section{Die Fermatsche Vermutung}

Wir erwähnen noch die Fermatsche Vermutung, die besagt, dass die Gleichung $a^{n}+b^{n}=$ $c^{n}$ in natürlichen Zahlen $a, b, c$ für $n>2$ unlösbar ist. Sie wurde erst 1994 durch A. Wiles [12] bewiesen.

Euler erwähnt diese Vermutung zuerst im Brief 125 vom 13. Februar 1748. Im Brief 169 vom 4. August 1753 schreibt er:

Bei Fermat findet sich noch ein sehr schönes theorema, dessen Demonstration er sagt, gefunden zu haben. Nämlich bei Anlass der Diophantaeischen Aufgabe, zwei quadrata zu finden, deren Summ ein Quadrat ist, sagt er, dass es unmöglich sei, zwei cubos zu finden, deren Summ ein Kubus sei, und zwei biquadrata, deren Summ ein biquadratum, und generaliter, dass diese Formul $a^{n}+b^{n}=c^{n}$ allzeit unmöglich sei, wann $n>2$. Ich habe nun wohl Demonstrationen gefunden, dass $a^{3}+b^{3} \neq c^{3}$ und $a^{4}+b^{4} \neq c^{4}$, wobei $\neq$ unmöglich gleich bedeutet. Aber die Demonstrationen für diese zwei casus sind so von einander verschieden, dass ich keine Möglichkeit sehe, daraus eine allgemeine Demonstration für $a^{n}+b^{n} \neq c^{n}$ si $n>2$ herzuleiten. 
Der Beweis im Falle $n=4$ war Fermat bekannt. Eulers Beweise für $n=3$ und $n=4$ findet man in seiner Algebra, siehe [10].

In den folgenden drei Abschnitten geben wir Beispiele bedeutender Ergebnisse von Euler, die zum Ausgangspunkt von Theorien wurden und zuerst im Briefwechsel mit Goldbach angesprochen wurden.

\section{Die Eulersche Identität}

Der oben erwähnte Philippe Naudé schrieb 1740 einen Brief an Euler, in dem er unter anderem die Frage stellte, was man über die Anzahl der Zerlegungen einer natürlichen Zahl $n$ in die Summe von $m$ natürlichen Zahlen sagen könne. Euler verstand sogleich, dass dies am besten mit der erzeugenden Funktion

$$
\prod_{i=1}^{\infty}\left(1+x^{i} z\right)=1+\sum_{n, m=1}^{\infty} S(n, m) x^{n} z^{m}
$$

ausgedrückt werden kann, wobei $S(n, m)$ die Anzahl der Darstellungen von $n$ als Summe von $m$ paarweise verschiedenen natürlichen Zahlen ist.

In diesem Zusammenhang kam er auf die Betrachtung des Produktes $\prod_{k=1}^{\infty}\left(1-x^{k}\right)$. Im Brief 74 vom 15. Oktober 1743 schreibt er, dass die Ausmultiplikation dieses Produktes die Reihe

$$
\sum_{j=-\infty}^{\infty}(-1)^{j} x^{(3 j-j) / 2}
$$

ergibt. Sämtliche Koeffizienten sind also von der Form - 1, 0 oder +1. Er schreibt dann:

Ich habe aber noch keine Methode finden können, wodurch ich die Identität dieser 2 Expressionen demonstrieren könnte.

Dieser Beweis findet sich im Brief 144 vom 9. Juni 1750.

Die vollständige Bedeutung der Eulerschen Identität ergab sich erst im 19. Jahrhundert im Rahmen der Theorie der Modulformen:

Die komplexe Funktion

$$
\eta(z)=q^{1 / 24} \prod_{k=1}^{\infty}\left(1-q^{k}\right), \quad q=e^{2 \pi i z},
$$

wird als Dedekindsche $\eta$-Funktion bezeichnet. Aus der Eulerschen Identität folgt unmittelbar

$$
\eta(z)=\sum_{j=1}^{\infty} \chi(j) q^{j^{2} / 24},
$$

wobei $\chi$ der Dirichlet-Charakter modulo 12 mit $\chi( \pm 1)=1, \chi( \pm 5)=-1$ ist.

Schon früher hatte Jacobi die Diskriminantenmodulform $\Delta(z)=(2 \pi)^{12} \eta(z)^{24}$ betrachtet. 


\section{Eine trigonometrische Reihe}

Im Brief 80 vom 4. Juli 1744 schreibt Euler:

Ich arbeite anjetzo an einem Traktat über den calculum differentialem, in welchem ich verschiedene kurieuse Decouverten über die series gemacht habe, wovon ich die Freiheit nehme, ew. Wohlgeboren einige zu kommunizieren.

Die erste dieser Decouverten ist die Gleichung

$$
\frac{\pi}{2}=\frac{a}{2}+\sum_{j=1}^{\infty} \frac{1}{j} \sin j a .
$$

Diese Gleichung erscheint auf den ersten Blick absurd, denn für $a=0$ ergibt sich $\frac{\pi}{2}=0$. Eine nach heutigen Maßstäben befriedigende Darstellung findet sich bei Dirichlet, [1]. Danach stellt die Reihe $\sum_{j=1}^{\infty} \frac{1}{j} \sin j a$ die periodische Funktion mit der Periode $2 \pi$ dar, die für $0<a<2 \pi$ durch $\frac{\pi}{2}-\frac{a}{2}$ und für $a=0$ durch 0, d.h. durch den Mittelwert der Sprungstelle, gegeben ist.

Euler gibt im Brief 80 die ersten Beispiele für eine Entwicklung einer Funktion in eine trigonometrische Reihe an, die später Fourier-Entwicklung genannt wurde.

\section{Eulerscher Polyedersatz}

Im Brief 149 vom 14. November 1750 schreibt Euler:

Neulich kam mir in den Sinn, die allgemeinen Eigenschaften der Körper, welche hedris planis eingeschlossen sind, zu bestimmen, weil kein Zweifel ist, dass sich in denselben nicht ebendergleichen allgemeine Eigenschaften finden sollten, als in den figuris planis rectilineis, .... Wie aber in den figuris planis nur latera und anguli zu betrachten vorkommen, müssen bei den Körpern mehr Stücke in Betrachtung gezogen werden.

Statt Körper, welche hedris planis eingeschlossen sind, sagen wir heute Polyeder, die zur Kugeloberfläche homöomorph sind. Mit figuris planis rectilineis sind Polygone gemeint.

Euler bezeichnet die Anzahl der Flächen des Polyeders mit $H$, die Anzahl der Kanten mit $A$ und die Anzahl der Ecken mit $S$. Er schreibt weiter:

Folgende Proposition aber kann ich noch nicht recht rigorose demonstrieren: $H+S=A+2$.

Dies ist der Eulersche Polyedersatz, den er 1758 mit Beweis publizierte [3]. Er kann als Ausgangspunkt der Disziplin betrachtet werden, die als kombinatorische Topologie bezeichnet wird. 
Goldbach antwortet im Brief 150 vom 4. Juni 1751:

Eurer Hochedelgebornen bin ich für die mir kommunizierten schönen theoremata von den Eigenschaften der Körper, welche hedris planis eingeschlossen sind, sehr verbunden, ich beklage aber, dass bei mir die gehörige Attention zu dergleichen Betrachtungen je länger je mehr und zwar per seriem valde ad nihilum convergentem wider meinen Willen abnimmt.

Goldbach bleibt aber interessiert am Vierquadratesatz.

\section{Der letzte Brief Eulers}

Der letzte Brief Eulers an Goldbach stammt vom 17. März 1764. Er begeistert sich darin für ein Ergebnis von J.H. Lambert (1728-1777), dem Begründer der Bayrischen Akademie der Wissenschaften. Lambert zeigt, dass die Gleichung $z^{n}=a z^{m}+1$ die Lösung

$$
z=1+\frac{a}{n}+\sum_{j=2}^{\infty} \frac{(j m-n+1) \cdots(j m-(j-1) n+1)}{j !} \cdot \frac{a^{j}}{n^{j}}
$$

hat. Euler schreibt:

Er hat mir eine seriem kommuniziert, darüber ich erstaunt bin, da dieselbe von einer ganz anderen Beschaffenheit ist, als alle diejenigen, so bisher betrachtet worden.

Eine Antwort von Goldbach auf diesen Brief ist nicht bekannt. Er starb am 1. Dezember 1764.

Damit beschließen wir unseren Einblick in den Briefwechsel Euler-Goldbach. Der gesamte Briefwechsel (mit Kommentaren) nimmt in der Publikation [6] 387 Seiten im DIN A4 Format ein.

\section{Literatur}

[1] Dirichlet, P.G.L: Sur la convergence des séries trigonométriques qui servent à représenter une fonction arbitraire entre des limites données. J. Reine Angew. Math. 4 (1829), 157-169.

[2] Euler, L.: Commentarii Academiae scientiarum Imperialis Petropolitanae 6 (1732-1733).

[3] Euler, L.: Novi commentarii Academiae scientiarum Imperialis Petropolitanae 6 (1752-1753).

[4] Fuss, P.H. (Hrsg.): Correspondance mathematique et physique de quelques célebrès géométres du XVIII $^{\text {éme }}$ siècle. Band 1, St. Petersburg 1843.

[5] Jushkevich, A.P.; Kopelevich, Ju.Kh.: Christian Goldbach 1690-1764. Verlag Nauka, Moskau 1983 (russisch).

[6] Jushkevich, A.P.; Winter, E. (Hrsg.): Leonhard Euler und Christian Goldbach. Briefwechsel 1729-1764. Akademie-Verlag, Berlin 1965.

[7] Lagrange, J.L.: Mémoires de l'Académie Royale des sciences et des belles lettres. Berlin 1768.

[8] Lagrange, J.L.: Nouvelles Mémoires de l'Académie Royale des sciences et des belles lettres. Berlin 1770. 
[9] Riemann, G.F.B.: Monatsberichte der Preußischen Akademie der Wissenschaften. November 1859.

[10] Schappacher, N.: xxxxxxxxxx. Elem. Math. 4 (2007), 133-xxx.

[11] Vinogradov, I.M.: Representation of an Odd Number as the Sum of Three Primes. Dokl. Akad. Nauk SSSR 15 (1937) 6-7, 191-194.

[12] Wiles, A.: Modular elliptic curves and Fermat's Last Theorem. Ann. of Math. 142 (1995), 443-551.

Helmut Koch

Humboldt-Universität zu Berlin

Institut für Mathematik

D-10099 Berlin, Deutschland

e-mail: koch@math.hu-berlin.de 